\title{
Gesundheitsstatistik: Perspektiven in der Schweiz?
}

\author{
F. Paccaud
}

In der Schweiz liegen im Berelch Gesundheitswesen eine grosse Zahl von Datensammlungen vor, die regelmässig von offentichen und privaten Institutionen erhoben werden (1). Dieser hohe Datenumfang bedeutet jedoch keineswegs, dass im Gesundheitswesen ein eigentiliches Informationssystem existiert, wie es die Weltgesundheitsorganisation definiert, nämlich : "ein Instrumentarium fux die Erhebung, Bearbeitung, Analyse und das Uebermitteln von fir die organisation und Verwaltung von Gesundheitsdiensten sowie für Forschung und Ausbildung ben'bitigten Informationen" (2).

Der Rijokstand der Schweiz in dieser Hinsicht ist offensichtlich, nicht nur mit Hinblick auf die erwähnte Definition sondern auch im Vergleich zu anderen industrialisierten Ländern. $E_{s}$ ist jedoch nicht sicher, dass diese Tatsache auch als beunruhigend exkannt wird : Weder durch vorschläge einer Arbeitsgruppe des Eidgenössischen Departements des Innern (3) noch durch gewisse Einzelinitiativen gelang es, eine ganzheitliche zukunftsperspektive für ale Entwicklung der Gesundheitsstatistik zu schaffen. Es gelang nicht einmal bei den betroffenen Berufszweigen ein Interesse für die Bedeutung dieses Problemkreises zu wecken.

Dieser Rijokgtand und aiese beständige Apathie können zum Teil der politischen, organisatorischen und ökonomischen Komplexität des schweizerischen Gesundheitssystems zugeschrieben werden. Sie zeugen jedoch auch von einer Unterschätzung der Rolle der Gesundheitsstatistik sowohl bei den verwaltungen im Gesundheitswesen als auch in wissenschaftlichen Kreisen.

In aen Tat nutzen viele verwaltungen im Gesundheitswesen diesbezúgliche Daten - die sie oft sogar selbst erheben - nur ungenügend, um nicht zu sagen iberhaupt nicht. Die statistiken scheinen manchmal nur zum ziel gewissenhafter Archivierung, der Herausgabe einer jährlichen Publikation oder für Illustrationszwecke in Zusammenhang mit gelegentlichen Anfragen erstellt worden zu sein.

Kurz, zu oft lässt sich die den Statistiken im Gesundheitswesen zugewiesene Rolle mit den Verhältnis leicht angeheiterter Nachtschwärmer zu Laternen vergleichen: "more for support than for illumination" (4). Solange diese Statistiken nicht als Datengrundlage fir Verwaltung und Planung genutzt und somit als ein wichtiges und kostbares Instrument erkannt werden, gibt es wenig Anlass zur Hoffnung, dass die Verwaltungen in Gesundheitswesen die nötige unterstitzung fïr die Bereitstellung eines adäquaten Informationssystems leisten werden.

Die wissenschaftichen Kreise ihrerseits stehen noch mit zu grossen Misstrauen den routinemässig bereitgestellten Daten gegenijber, die sie als wenig glaubwirdig und/oder ungeeignet als Grundlage für ihre For- schungen exachten. Die offensichtlichen Grenzen bei der Nutzung gewisser Daten sollten jedoch einer sorgfältigen Prufung und Bewertung der gegebenen Möglichkeiten durch bastehende Erhebungen nicht im Wege stehen, umsomeht wenn als einzige Alternative die Durchführung einer spezialerhebung stehen wïrde, was immer mit elnem hohen Aufwand an Kosten und zeit verbunden ist. Eine intensive und ideenreiche Analyse von schon bestehenden statistiken kann die Datenproduzenten auf die Nitzlichkeit ihrer Erhebung hinweisen und sie so ermutigen ein befriedigendes Qualitätsniveau au halten. Längerfristig ist eine gute offizielle statistik eine unabdingbare Voraussetzung für Forschungstätigkeit : das hohe Niveau epiderniologischer Studien im angelsäxischen und skandinavischen Raum erklärt sich nicht zuletzt durch die inhaltliche Fỉlle der Routineerhebungen.

Als bescheidenes Plädoyer für die Gesundheitsstatistik. werden in dieser Nummer einige Arbeiten vorgestellt, wo Medizin-statistische Daten verwendet oder kommentlert wexden, die von offentlichen Stellen routinemässig erhoben werden. Die Mehrzahl aer Artikel. befasst sich mit der Mortalität, un zeugnis von der bestehenden Aktualitat einex der ersten von der Epidemiologie ausgewerteten statistiken zu geben. Drei Beiträge befassen sich mit Datenerhebungen im Bereich Morbiaität.

Ein abschliessender Artikel behandelt das Thema Datenschutz, ohne dessen Beriicksichtigung jede Betrachtung im Bereich Gesundheitsstatistik unvollständig wäre. Dieser Beitrag zeigt mit Klarheit die verschiedenen Positionen und Rahmenbedingungen, wie sie von den Juristen und dariberhinaus bestimmt auch von einer misstrauischen öffentlichen Meinung gesehen werden.

Herzlichen Dank gebuhrt hier allen beteiligten Autoren. Die Edition dieser Numer wurde möglich durch die Zusammenarbeit von Antoinette Rochat und Brigitte Bisig (Bunderamt fïr Statistik, Bern) und Mireille Christen (Institut universitaire de médecine sociale et préventive, Lausanne).

\section{Referenzen}

1. Schweizerisches Xrankenhausinstitut S.X.I./Institut für Sozial- und Präventivmedizin der Universität Bern. Register über Gesundheitsstatistiken, Aarau, S.K.I., 1981.

2. World Health Organization. Health Information Systems : Report on a conference, Copenhagen, W.H.O., 1973.

3. Bericht iber die Gesundheitsstatistik in der Schweiz, Bern, Eidgenössisches Departement der Innern, 1978 .

4. Silvermann W.A. Fetal heart rate monitoring, Lancet, 2 : 908,1979 . 